\title{
17beta-estradiol promotes the odonto/osteogenic differentiation of stem cells from apical papilla via mitogen-activated protein kinase pathway
}

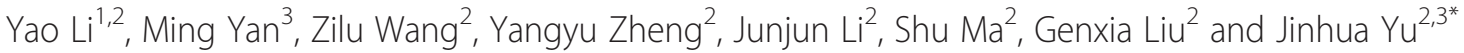

\begin{abstract}
Introduction: Estrogen plays an important role in the osteogenic differentiation of mesenchymal stem cells, while stem cells from apical papilla (SCAP) can contribute to the formation of dentin/bone-like tissues. To date, the effects of estrogen on the differentiation of SCAP remain unclear.

Methods: SCAP was isolated and treated with $10^{-7} \mathrm{M} 17$ beta-estradiol (E2). The odonto/osteogenic potency and the involvement of mitogen-activated protein kinase (MAPK) signaling pathway were subsequently investigated by using methyl-thiazolyl-tetrazolium (MTT) assay, and other methods.

Results: MTT and flow cytometry results demonstrated that E2 treatment had no effect on the proliferation of SCAP in vitro, while alkaline phosphatase (ALP) assay and alizarin red staining showed that E2 can significantly promote ALP activity and mineralization ability in SCAP. Real-time reverse transcription polymerase chain reaction (RT-PCR) and western blot assay revealed that the odonto/osteogenic markers (ALP, DMP1/DMP1, DSPP/DSP, RUNX2/ RUNX2, OSX/OSX and OCN/OCN) were significantly upregulated in E2-treated SCAP. In addition, the expression of phosphor-p38 and phosphor-JNK in these stem cells was enhanced by E2 treatment, as was the expression of the nuclear downstream transcription factors including phosphor-Sp1, phosphor-Elk-1, phosphor-c-Jun and phosphor-c-Fos, indicating the activation of MAPK signaling pathway during the odonto/osteogenic differentiation of E2-treated SCAP. Conversely, the differentiation of E2-treated SCAP was inhibited in the presence of MAPK specific inhibitors.
\end{abstract}

Conclusions: The ondonto/osteogenic differentiation of SCAP is enhanced by $10^{-7} \mathrm{M} 17$ beta-estradiol via the activation of MAPK signaling pathway.

\section{Introduction}

When the dental pulp of a young tooth with open apex is infected due to caries or trauma, dentists often try to take measures (for example, apexification and apexogenesis) to preserve the root development and maturation [1]. During the process, a series of factors are involved in the growth of the root, for instance, stem cells from apical papilla (SCAP), which still exist after the formation of the root and can survive the infection [2,3]. SCAP have a high proliferation rate and possess osteo/dentinogenic and adipogenic potentials $[4,5]$. When transplanted into

\footnotetext{
* Correspondence: yujinhua@njmu.edu.cn

${ }^{2}$ Key Laboratory of Oral Diseases of Jiangsu Province and Stomatological Institute of Nanjing Medical University, 140 Hanzhong Road, Nanjing, Jiangsu 210029, China

${ }^{3}$ Endodontic Department, School of Stomatology, Nanjing Medical University, 136 Hanzhong Road, Nanjing, Jiangsu 210029, China

Full list of author information is available at the end of the article
}

immunocompromised mice, SCAP can form a typical dentin-pulp-like complex and generate bone-like tissues containing osteoblast-like cells [4-6]. Moreover, the combination of SCAP and periodontal ligament stem cells (PDLSCs) can produce dentin and cementum with collagen fibers anchored into the cementum after transplantation in vivo, which represents an approach to biological root engineering [7]. Thus, such a biological root supporting a porcelain crown can restore a missing tooth instead of bridges and removable dentures. Huang et al. [2] have suggested that SCAP play an important role in root formation. Many factors can affect the proliferation and odonto/osteogenic differentiation of SCAP, including culture medium, cell phenotype, medicaments, growth factors, hormones, morphogens, and so on [8-11] that demonstrate the importance of the extrinsic microenvironment to SCAP when applied in tissue engineering. 
Hormones are very important to the growth, development, reproduction and maintenance of a diverse range of mammalian tissues. Estrogen is known as one of the important hormones for sex maturation and bone metabolism [12]. Previous research has revealed a close relationship between estrogen deficiency and osteoporosis that occurs in postmenopausal women, and older men as well, leading to decreased bone mineral density and even bone fracture [13]. It has also been demonstrated that periodontal diseases are related to estrogen deficiency which causes impaired osteogenic differentiation of PDLSCs [14]. Furthermore, an in vivo study has shown that the differentiation ability of dental pulp stem cells (DPSCs) was downregulated in estrogen deficient rats [12]. Recent studies have suggested that exogenous estrogen can enhance the proliferation and differentiation of bone marrow mesenchymal stem cells (BMMSCs), PDLSCs and DPSCs $[8,15,16]$. To date, the effects of estrogen on SCAP remain unclear.

In this study, we investigated the influence of estrogen on the proliferation and odonto/osteogenic differentiation of SCAP in vitro. SCAP was isolated from extracted third molars and exposed to 17beta-estradiol (E2) [17]. Then, the proliferation, differentiation and involvement of the MAPK signaling pathway in E2-treated SCAP were determined. Our findings suggest that E2 can enhance the odonto/osteogenic differentiation of SCAP via the MAPK pathway.

\section{Methods}

\section{Cell isolation and culture}

Impacted non-carious third molars $(\mathrm{n}=20)$ were collected from young patients (17- to 20-years old) in the Oral Surgery Department of Jiangsu Provincial Stomatological Hospital. This study was approved by the Ethical Committee of the Stomatological School of Nanjing Medical University (Reference \#200900128), and consent from patients was obtained. The apical papillae were carefully separated from the immature roots, minced and digested in a solution containing $3 \mathrm{mg} / \mathrm{ml}$ collagenase type I (Sigma, St. Louis, $\mathrm{MO}, \mathrm{USA}$ ) and $4 \mathrm{mg} / \mathrm{ml}$ dispase (Sigma) for 30 minutes at $37^{\circ} \mathrm{C}$. Single cell suspensions were obtained and cells from different patients were mixed. Isolated cells were cultured in alpha minimum essential medium ( $\alpha$-MEM, Gibco, Life Technologies, Grand Island, NY, USA) supplemented with $10 \%$ fetal bovine serum (FBS, Hyclone, Logan, UT, USA), $100 \mathrm{U} / \mathrm{mL}$ penicillin and $100 \mathrm{mg} / \mathrm{mL}$ streptomycin at $37^{\circ} \mathrm{C}$ in $5 \% \mathrm{CO}_{2}$. 17beta-estradiol (E2, Ehrenstorfer Gmbh, Augsburg, Germany) was dissolved in absolute ethyl alcohol at $10^{-3} \mathrm{M}$ and stored at $-20^{\circ} \mathrm{C}$ in the dark. In the subsequent experiments, cells were cultured in $\alpha$-MEM containing E2 (E2 group) or $0.01 \%(\mathrm{v} / \mathrm{v})$ ethyl alcohol as control (control group). The culture medium was changed every two days. JNK and p38 specific inhibitors SP600125 (Sigma-Aldrich,
St. Louis, MO, USA) and SB203580 (Sigma-Aldrich) were dissolved in dimethyl sulfoxide (DMSO, Sigma-Aldrich) at $100 \mathrm{mM}$ and $20 \mathrm{mM}$, respectively and stored at $-20^{\circ} \mathrm{C}$ in the dark. They were diluted to $20 \mu \mathrm{M}$ when added to the culture media. Cells at passages two to four were used for the following experiments.

\section{Cell identification}

To determine the origin of the obtained mesenchymal stem cells, isolated cells were immunostained with antibody against STRO-1 (1:200, Novus Biologicals, Littleton, CO, USA) and their surface markers were measured by flow cytometry. Cells were harvested and incubated with fluorochrome-conjugated rabbit anti-human antibodies: CD34-FITC, CD45-PerCP, CD90-PE, CD105-APC, CD146APC, CD73-PE (Miltenyi, Bergisch Gladbach, Germany) for 20 minutes at room temperature in the dark. Stained cells were washed twice with $0.01 \mathrm{M}$ phosphate buffer solution (PBS) and analyzed by BD FACSCalibur (BD Biosciences, San Jose, CA, USA).

\section{In vivo transplantation}

The animal experiments were approved by the Ethical Committee of the Stomatological School of Nanjing Medical University. SCAP $\left(1 \times 10^{6}\right)$ was collected as a pellet in a sterile tube and seeded gently onto absorbable gelatin sponges (AGS, Nanjing Pharmaceuticals, Nanjing, China), which served as carriers. Then, cell pellets were transplanted into the renal capsules of immunodeficient female rats. After in vivo culture for eight weeks, the retrieved implants $(\mathrm{n}=6)$ were fixed in $4 \%$ polyoxymethylene, decalcified and processed for hematoxylin and eosin (H \& E) staining.

\section{Immunohistochemistry and immunocytochemistry}

Immunohistochemical and immunocytochemical analyses of human tissues or human SCAP were performed by the streptavidin-biotin complex method using the primary antibodies (STRO-1, 1:200, Santa Cruz, Dallas, TX, USA; ER- $\alpha, 1: 100$, Abcam, Cambridge, UK) according to the manufacturers' recommended protocols $[18,19]$. The reaction products were developed in 3, 3'-diaminobenzidine solution with hydrogen peroxide and counterstained with hematoxylin.

\section{MTT assay}

SCAP were seeded into 96-well plates (Nunc, Thermo Scientific, Waltham, MA, USA) at a density of $2 \times 10^{3}$ cells/ well for 24 hours and starved in a serum-free medium for another 24 hours. Then the medium was changed to complete medium containing E2. At different time points (days $0,1,3,5,7,9$ and 11), the cells were treated with MTT (3-[4, 5-dimethylthiazol-2-yl]-2, 5-diphenyl-2, 5tetrazoliumbromide) solution (5 $\mathrm{mg} / \mathrm{ml}$; Sigma-Aldrich) and incubated at $37^{\circ} \mathrm{C}$ for four hours. Then, the solution 
was removed and $150 \mu \mathrm{l} /$ well DMSO was added. The absorbance (OD value) was measured at $490 \mathrm{~nm}$ with an automatic enzyme-linked immunosorbent assay reader (ELx800, BioTek Instruments Inc., Grand Island, NY, USA). The experiment was repeated three times and MTT results are expressed as the mean \pm SD.

\section{Colony forming assay}

SCAP in the control group and the E2 group were seeded into six-well plates (Nunc, USA) at a density of $1 \times 10^{2}$ cells/well for two weeks. Then, the cells were fixed with $4 \%$ paraformaldehyde (PFA), stained with crystal violet (Beyotime, Shanghai, China) and photographed. The colonies were visualized under an inverted microscope (Olympus, Hamburg, Germany). Aggregations of more than 50 cells were defined as colonies and then counted. The experiment was repeated three times.

\section{Flow cytometry for cell cycle}

SCAP were plated into 6-cm culture dishes (Nunc, USA), cultured in $\alpha$-MEM supplemented with 10\% FBS until 60\% to $70 \%$ confluence, and then serum-starved for 24 hours. E2 was added to the culture media of the experimental groups. After three days of incubation, the cells were harvested and fixed with $75 \%$ ice-cold ethanol at $4^{\circ} \mathrm{C}$ for 30 minutes in the dark. DNA content was measured by FAC-Scan flow cytometer (BD Biosciences, San Jose, CA, USA). Cell cycle fractions $\left(G_{0} / G_{1}, S\right.$, and $G_{2} / M$ phases $)$ were determined by flow cytometry (FCM). The experiment was repeated three times.

\section{Alkaline phosphatase (ALP) activity assay and alizarin red staining}

SCAP in the control group and the E2 group were seeded into 96-well plates (Nunc, USA) at a density of $2 \times 10^{3}$ cells/well or 24 -well plates (Nunc, USA) at a density of $1 \times 10^{4}$ cells/well and cultured in routine media or mineralization-inducing media $(\mathrm{MM})$ containing $\alpha$-MEM, 10\% FBS, $100 \mathrm{U} / \mathrm{ml}$ penicillin, $100 \mu \mathrm{g} / \mathrm{ml}$ streptomycin, $100 \mu \mathrm{M}$ ascorbic acid, $2 \mathrm{mM}$ 2-glycerophosphate and $10 \mathrm{nM}$ dexamethasone. Alkaline phosphatase (ALP) activity assay was performed as previously reported [20] by using an ALP activity kit (Sigma-Aldrich) and normalized to total protein content in the cells at days 5 and 7 . At day 14, alizarin red staining was carried out as described before [21] and images were acquired using a scanner. Then, nodule staining was destained by $10 \%$ cetylpyridinium chloride (CPC) in $10 \mathrm{mM}$ sodium phosphate for 30 minutes at room temperature. The calcium concentration was determined by measuring the absorbance at $526 \mathrm{~nm}$ with a universal microplate reader (BioTek Instruments). This experiment was performed in triplicate and the results are presented as the mean \pm SD.
Real-time reverse transcription polymerase chain reaction (real-time RT-PCR)

Total cell RNA was isolated using TRIzol reagent (Invitrogen, New York, NY, USA) according to the manufacturer's protocol. The concentration and purity of the RNA samples were determined by the absorbance of RNA at 230, 260 and $280 \mathrm{~nm}$, respectively. The mRNA was reversetranscribed into cDNA by using a PrimeScript RT Master Mix kit (TaKaRa Biotechnology, Dalian, China). Real-time RT-PCR was performed using a SYBR1 Premix Ex Taq kit (TaKaRa, Otsu, Japan) and ABI 7300 real-time PCR system. Real-time RT-PCR reaction conditions were: $95^{\circ} \mathrm{C}$ for 30 seconds; followed by 40 cycles of $95^{\circ} \mathrm{C}$ for 5 seconds, $60^{\circ} \mathrm{C}$ for 31 seconds. Primers used in this experiment are listed in Table 1. GAPDH was used as an internal control and the expression of osteo/odontoblast-associated genes (ALP, DSPP, DMP1, RUNX2, OSX and OCN) was measured by the $2^{-\Delta \Delta \mathrm{Ct}}$ method as previously reported. Data are given as the mean \pm SD of three independent experiments.

\section{Western blot analysis}

To explore the effects of E2 on odonto/osteogenic differentiation of SCAP, SCAP in the control group and the E2 group were cultured for three and seven days, respectively, and then collected. For the evaluation of the MAPK signaling pathway, SCAP was seeded on $6-\mathrm{cm}$ dishes. After 24 hours of incubation, cells were serumstarved for another 24 hours before exposure to E2 for 30 minutes, 60 minutes and 90 minutes, respectively. Cells in different groups were washed twice with cold PBS and lysed in radioimmunoprecipitation assay (RIPA) lysis buffer (Beyotime) containing $1 \mathrm{mM}$ phenylmethylsulfonyl fluoride (PMSF). Cell debris was eliminated by

Table 1 Sense and antisense primers for real-time reverse transcription polymerase chain reaction

\begin{tabular}{|c|c|c|}
\hline Genes & Primers & Sequences $\left(5^{\prime}-3^{\prime}\right)$ \\
\hline \multirow[t]{2}{*}{$\overline{A L P}$} & Forward & GACCTCCTCGGAAGACACTC \\
\hline & Reverse & TGAAGGGCTTCTTGTCTGTG \\
\hline \multirow[t]{2}{*}{ DSPP } & Forward & ATATTGAGGGCTGGAATGGGGA \\
\hline & Reverse & TTTGTGGCTCCAGCATTGTCA \\
\hline \multirow[t]{2}{*}{$D M P 1$} & Forward & CCCTTGGAGAGCAGTGAGTC \\
\hline & Reverse & CTCCTITTCCTGTGCTCCTG \\
\hline \multirow[t]{2}{*}{$R \cup N \times 2$} & Forward & TCTTAGAACAAATTCTGCCCTIT \\
\hline & Reverse & TGCTITGGTCTTGAAATCACA \\
\hline \multirow[t]{2}{*}{ OSX } & Forward & ССTCCTCAGCTCACCTTCTC \\
\hline & Reverse & GTTGGGAGCCCAAATAGAAA \\
\hline \multirow[t]{2}{*}{ OCN } & Forward & AGCAAAGGTGCAGCCTITGT \\
\hline & Reverse & GCGCCTGGGTCTCTTCACT \\
\hline \multirow[t]{2}{*}{ GAPDH } & Forward & GAAGGTGAAGGTCGGAGTC \\
\hline & Reverse & GAGATGGTGATGGGATTTC \\
\hline
\end{tabular}


centrifugation at 12,000 rpm for 15 minutes. The cytoplasm protein and nucleoprotein were obtained with a Keygen Kit (Keygen Biotech, Nanjing, China). Protein concentration was measured by the Bradford protein assay. A total of $30 \mu \mathrm{g}$ protein per lane was loaded onto a 10\% SDS-PAGE gel for electrophoresis and then electroblotted (Bio-Rad, Hercules, CA, USA) onto $0.22 \mu \mathrm{m}$ polyvinylidene fluoride (PVDF) membrane (Millipore, Bedford, MA, USA) at $300 \mathrm{~mA}$ for one hour. Membranes were blocked with blocking solution ( $5 \%$ non-fat dried skimmed milk powder, 0.01 M PBS, 0.1\% Tween-20 (PBST)) at room temperature for two hours, and subsequently incubated with primary antibodies (DMP1, 1:1000, Abcam; DSP, 1:1000, Santa Cruz; RUNX2, 1:1000, Abcam; OSX, 1:1000, Abcam; OCN, 1:1000, Millipore, Billerica, MA, USA; ERK1/2, 1:1000, Bioworld , St. Louis Park, MN, USA; phosphor-ERK1/2, 1:1000, Bioworld; JNK1/2/3, 1:1000, Bioworld; phosphor-JNK1/2/3, 1:1000, Bioworld; p38, 1:1000, Bioworld; phosphor-p38, 1:1000, Bioworld; Elk-1, 1:1000, Cell Signaling, Danvers, MA, USA; phosphor-Elk-1, 1:1000, Cell Signaling; Sp1, 1:1000, Cell Signaling; phosphor-Sp1, 1:1000, Cell Signaling; c-Jun, 1:1000, Cell Signaling; phosphor-c-Jun, 1:1000, Cell Signaling; c-Fos, 1:1000, Cell Signaling; phosphor-c-Fos, 1:1000, Cell Signaling; ER- $\alpha, 1: 1000$, Abcam; $\beta$-ACTIN, 1:1000, Bioworld; H3, 1:1000, Bioworld) overnight at $4^{\circ} \mathrm{C}$. $\beta$-ACTIN and $\mathrm{H} 3$ were, respectively, used as the internal controls. Finally, the membranes were washed with PBST for 10 minutes $\times 3$ followed by incubation in secondary antibodies (1:10,000, Boster, Wuhan, China) for one hour at $37^{\circ} \mathrm{C}$, and visualized with ImageQuant LAS4000 system (GE Healthcare, Pittsburgh, PA, USA). The results were quantified with Image software (National Institutes of Health, Bethesda, MD, USA). The experiment was repeated three times.

\section{Immunofluorescence}

E2 treated and untreated SCAP were, respectively, cultured on glass coverslips. After three days culture, cells were washed twice with PBS, fixed in 4\% polyoxymethylene for 30 minutes at room temperature, permeabilized with $0.5 \%$ Tween 20 for 10 minutes and then blocked with goat serum for 30 minutes at $37^{\circ} \mathrm{C}$. After that, cells were incubated with primary antibodies (DSP, 1:50, Santa Cruz; RUNX2, 1:100, Abcam; OCN, 1:100, Millipore) overnight at $4^{\circ} \mathrm{C}$. The fluorescence-labeled secondary antibody was added and incubated for one hour at room temperature. Nuclei were stained by 4,6-diamidino-2-phenylindole (DAPI; 1:1,000; Invitrogen) for two minutes. Immunofluorescence was visualized under a microscope.

\section{Statistics}

The quantitative results were expressed as the mean \pm SD. Independent samples $t$ test, Chi-square test, one-way analysis of variance (ANOVA) and Tukey's multiple comparison test were performed with SPSS 17.0 software. $P$ values $<0.05$ were considered to be statistically significant.

\section{Results}

\section{Identification of SCAP}

The isolated cells presented the typical fibroblast- or spindle-like morphology and stained positively for STRO-1 (a mesenchymal stem cell marker, Figure 1A). FCM findings revealed that these cells were positive for CD73, CD146, CD90 and CD105, but negative for CD45 and CD34, indicating that these cells are mesenchymal stem cells (Figure 1C). Moreover, in vivo transplantation of such purified cells demonstrated that these cells formed the typical dentin-pulp-like complex containing the structures of dental pulp, odontoblasts, predentin and dentin (Figure 1D). Columnar odontoblasts with polarized nuclei aligned regularly along the predentin (Figure 1E).

\section{Dose-dependent effects of E2 on SCAP}

SCAP were treated with different concentrations of E2 $\left(10^{-9} \mathrm{M}, 10^{-7} \mathrm{M}\right.$, and $\left.10^{-5} \mathrm{M}\right)$ to detect the dose-dependent effects of E2. MTT results at day 5 demonstrated that $10^{-9} \mathrm{M}$ and $10^{-7} \mathrm{M} \mathrm{E} 2$ did not affect the proliferation of SCAP while $10^{-5} \mathrm{M}$ E2 significantly inhibited cell proliferation (Figure $2 \mathrm{~A} ; P<0.05$ ). ALP activity assay at day 5 revealed that $10^{-9} \mathrm{M}$ E2 had no effect on the differentiation of SCAP while $10^{-7} \mathrm{M}$ E2 significantly promoted cell differentiation (Figure 2B; $P<0.01$ ). However, the differentiation ability significantly decreased in $10^{-5} \mathrm{M}$ E2-treated SCAP (Figure 2B; $P<0.01$ ). Thus, $10^{-7} \mathrm{M}$ was selected as the optimal concentration of E2 used in the following experiments.

\section{Effects of E2 on morphology and proliferation of SCAP}

SCAP were cultured in $\alpha$-MEM containing $10^{-7} \mathrm{M}$ E2 (E2 group) or $0.01 \%(\mathrm{v} / \mathrm{v})$ ethyl alcohol as control (control group). Both the control group and the E2 group presented a spindle-like morphology and shared almost the same appearance (Figure 2C-F). FCM analysis showed no distinct difference $(P>0.05)$ in the proliferation index $(\mathrm{PI}=\mathrm{G} 2 \mathrm{M}+\mathrm{S})$ between the control group $(6.67 \%)$ and the E2 group (7.37\%) (Figure 2G-I). MTT assay on 11 consecutive days also presented no significant difference $(P>0.05)$ between the two groups (Figure $2 J)$, and neither did the results of a colony forming assay between the control group and the E2 group (Figure $2 \mathrm{~K}$ and L). E2 had no significant effect on the morphology and proliferation of SCAP.

Effects of E2 on odonto/osteogenic differentiation of SCAP Immunocytochemical analysis of SCAP showed that the staining of STRO-1 in the cytoplasma of E2-treated SCAP was weaker than that in control group, suggesting the downregulation of STRO-1 following the differentiation of E2-treated SCAP (Figure 3A and B). 

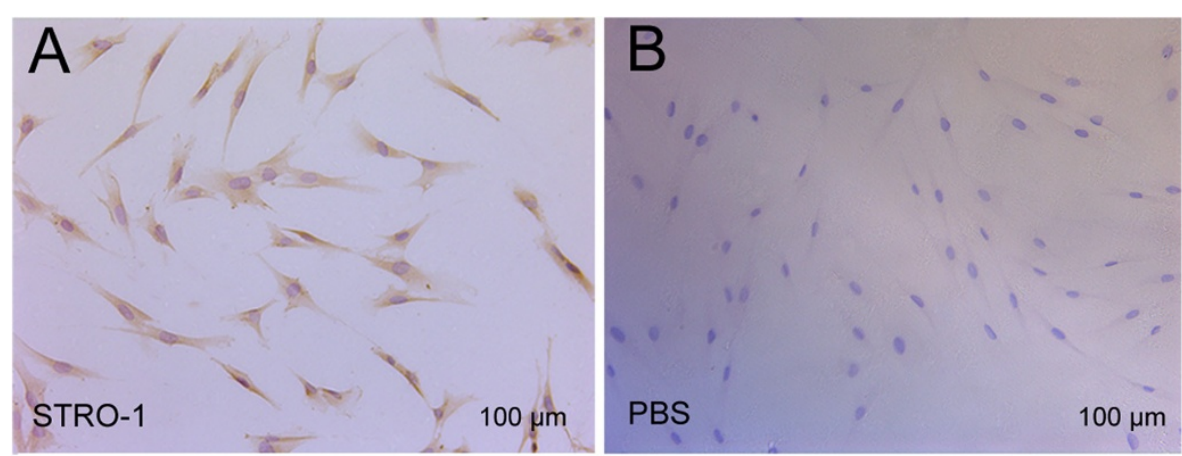

C
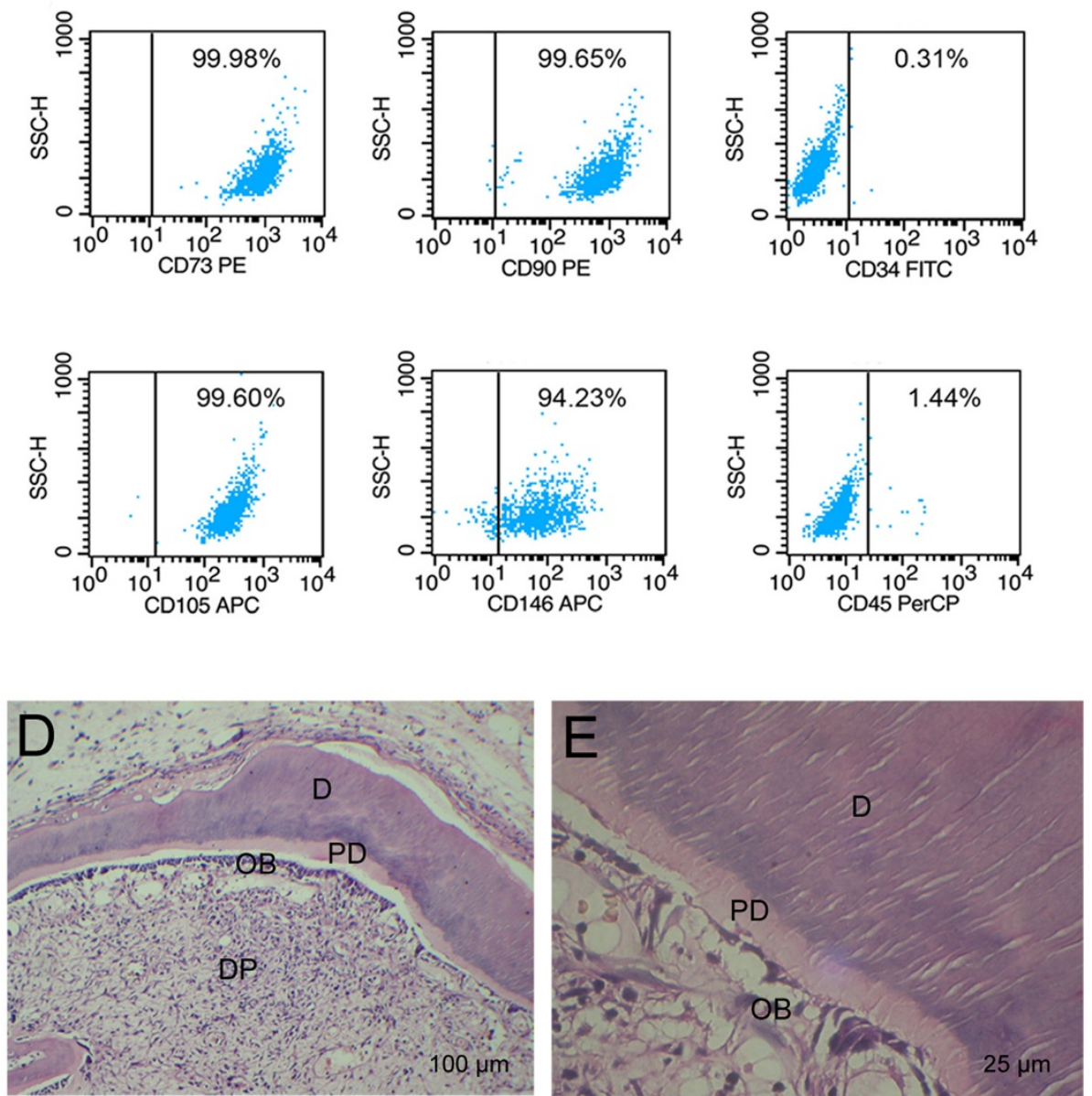

Figure 1 Identification of SCAP. (A) Isolated SCAP were stained positively for STRO-1 with typical fibroblast- or spindle-like morphology. (B) PBS served as a negative control. (C) Isolated SCAP were positive for CD73, CD146, CD90 and CD105, but negative for CD45 and CD34 by flow cytometry. (D) In vivo transplantation of SCAP pellets formed the typical dentin-pulp-like complex containing dental pulp, odontoblasts, predentin and dentin. (E) A higher magnification of (D). DP, dental pulp; OB, odontoblast; D, dentin; PD, predentin. Scale bars: $A, B$ and $D=100 \mu m$, $\mathrm{E}=25 \mu \mathrm{m}$. SCAP, stem cells from apical papilla.

SCAP were cultured in routine media or MM with or without E2. At day 5, E2 exerted no influence on ALP activity of SCAP in routine media, while ALP activity was noticeably upregulated by E2 in MM (Figure 3C; $P<0.01)$. At day 7 , both E2-treated groups exhibited a significant increase in ALP activity of SCAP (Figure 3C;
$P<0.01)$. Moreover, alizarin red staining and quantitative calcium measurement demonstrated that SCAP treated with E2 produced more calcium nodules after 14 days of incubation (Figure 3D and E; $P<0.05$ ). When observed under an inverted microscope, the calcium nodules formed in routine media were fewer and smaller, 


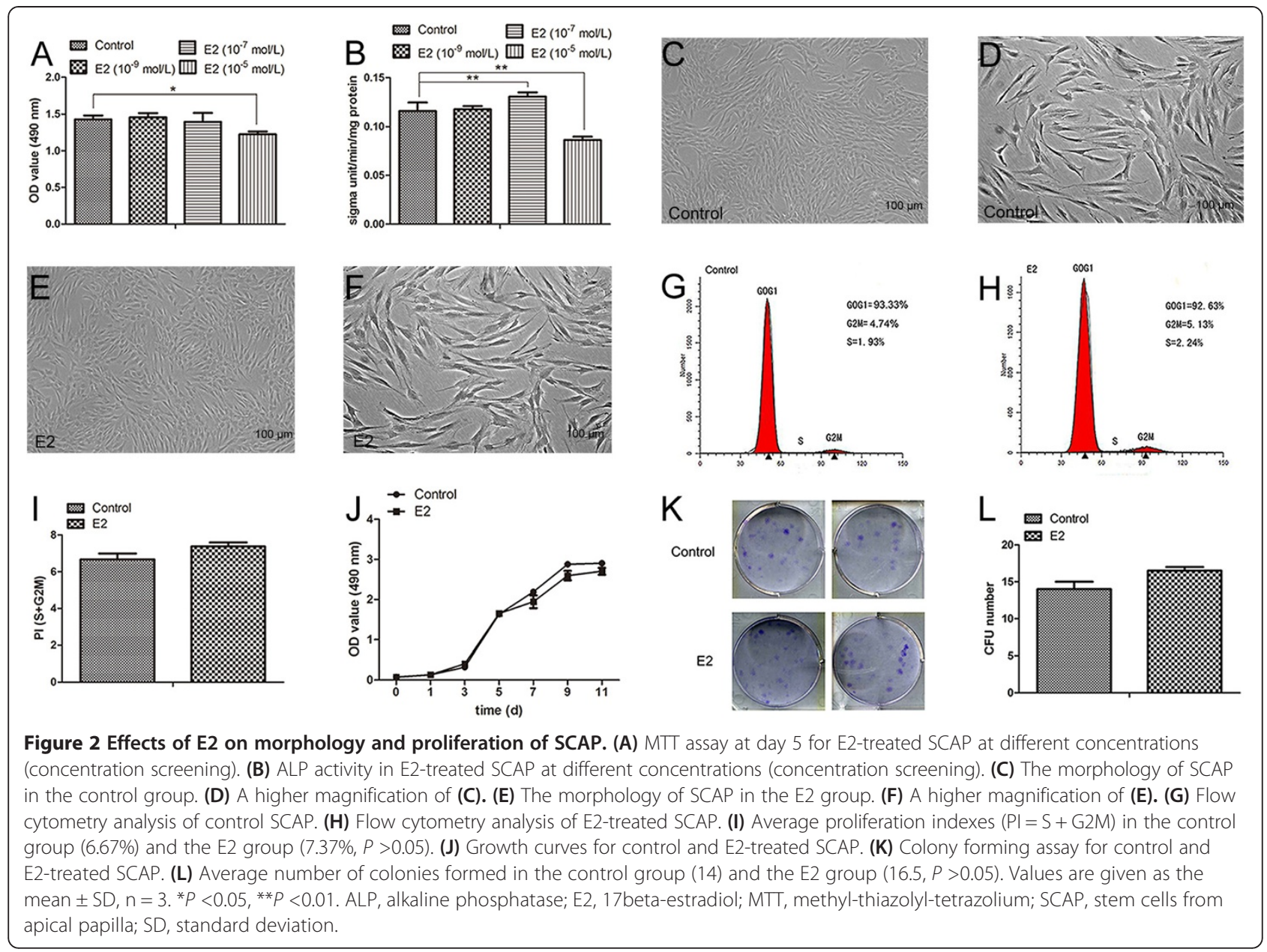

whereas SCAP generated more and larger calcium nodules in MM. Moreover, the shape of the calcium nodules in the $\mathrm{MM}+\mathrm{E} 2$ group was more regular than in other groups (Figure 3F-I).

Real-time RT-PCR showed that the odonto/osteogenic genes (ALP, DMP1, DSPP, RUNX2, OSX and OCN) were remarkably enhanced in E2-treated SCAP. In particular, the expression of ALP, DMP1, DSPP, RUNX2 and OSX increased from day 3 to day 7 at a significant extent (Figure 3J and $\mathrm{K} ; P<0.01$ ), while the expression of $O C N$ rose at a similar level at day 3 and day 7 (Figure 3J and $\mathrm{K} ; \mathrm{P}<0.05)$. Western blot findings showed that the expression of the odonto/osteogenic proteins (DMP1, DSP, RUNX2, OSX and OCN) was significantly upregulated in E2-treated SCAP at day 3 and 7, in comparison with cells in the control group (Figure 3L-O; $P<0.01$ or $P<0.05$ ).

The immunofluorescent results demonstrated that RUNX2 was expressed in the nuclei while OCN and DSP were mainly located in the cytoplasm. They were all enhanced following E2 treatment, indicating the promotion of odonto/osteogenic differentiation in SCAP by E2 (Figure 4).
Involvement of the MAPK pathway during differentiation of E2-treated SCAP

To determine the potential involvement of the MAPK signaling pathway in the E2-mediated differentiation of SCAP, we investigated the ER- $\alpha$ level as well as the expression of MAPK-related proteins and downstream transcription factors. SCAP showed positive staining for ER- $\alpha$ (Figure 5A) and the expression of ER- $\alpha$ was notably enhanced following E2 treatment (Figure 5D and E; $P<0.01$ ). However, E2 treatment did not affect the expression of total ERK, JNK and p38. The level of phosphor-ERK almost did not change with time, whereas phosphorylation of JNK and p38 gradually upregulated from 0 to $90 \mathrm{mi}-$ nutes following E2 treatment (Figure 5F-I; $P<0.01$ ). For the nuclear downstream transcription factors, the levels of total Sp1, Elk-1, c-Jun and c-Fos did not change in a timedependent manner. Phosphorylation of $\mathrm{Sp} 1$ was significantly enhanced at 60 minutes and remained at a high level at 90 minutes after E2 treatment (Figure 5J and K; $P<0.01)$. Phosphorylation of Elk-1 gradually increased from 0 to 60 minutes but was noticeably downregulated at 90 minutes by E2 treatment (Figure 5J and L; $P<0.01$ ). 


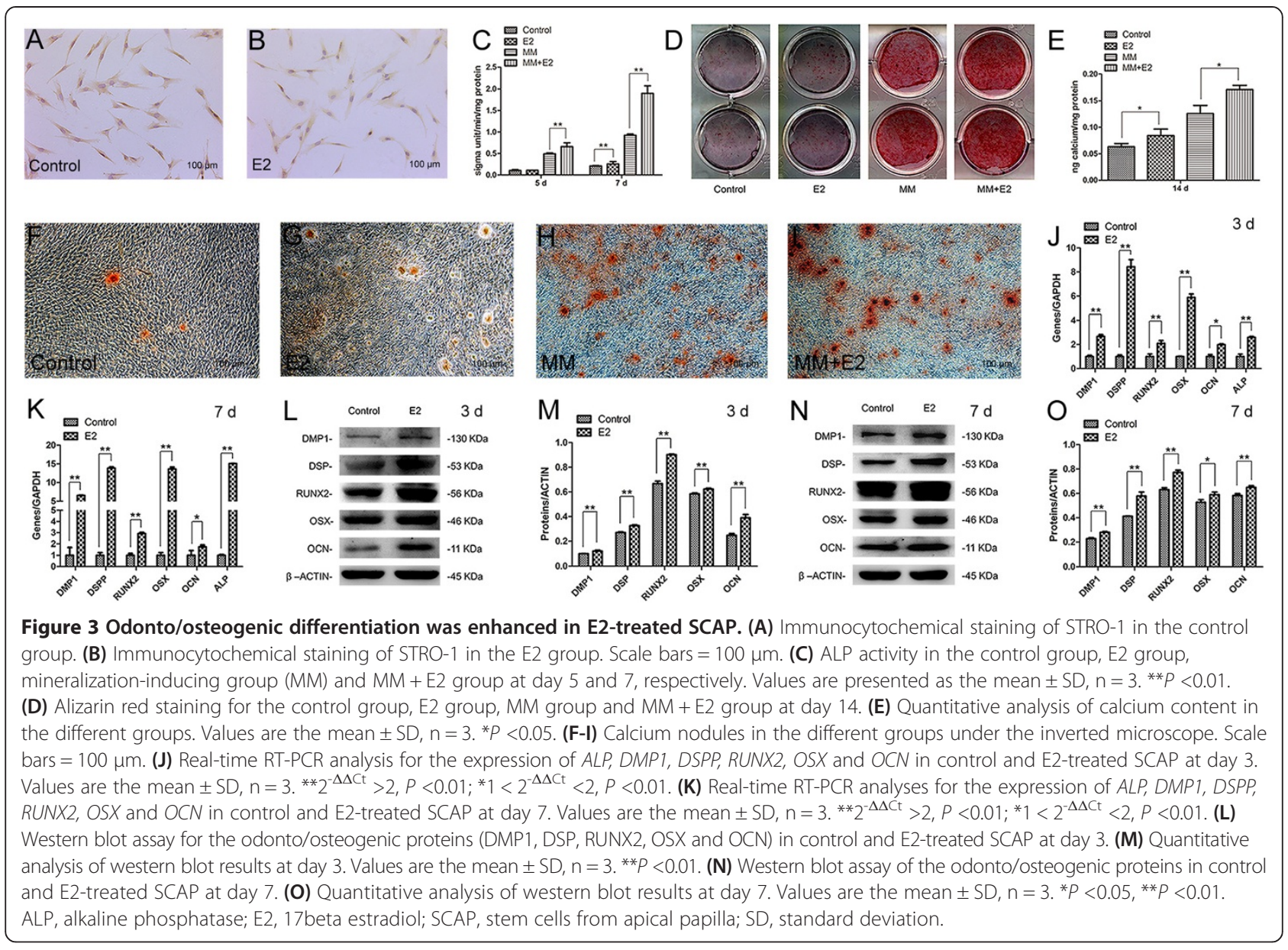

In addition, the level of phosphor-c-Jun was significantly upregulated at 30 minutes and then gradually decreased from 60 to 90 minutes following E2 treatment (Figure 5J and M; $P<0.01$ ). Moreover, the phosphor-c-Fos level rose from 0 to 90 minutes after treatment with E2 (Figure 5J and N; $P<0.01$ or $P<0.05)$.

To further determine the role of the MAPK signaling pathway during the differentiation of E2-treated SCAP, SP600125 and SB203580 (specific inhibitors of JNK and p38) were used at a concentration of $20 \mu \mathrm{M}$ to suppress the activity of JNK and p38 signaling for one hour prior to E2 treatment. The ALP activity of SCAP at day 5 was remarkably inhibited by SP600125 and SB203580 (Figure 6A; $P<0.01$ ). Furthermore, odonto/osteogenic proteins (DMP1, DSP, RUNX2, OSX and OCN) were significantly downregulated in the E2 + SP600125 group and the E2 + SB203580 group, in comparison with the E2 group at day 3 and day 7 (Figure 6B-E; $P<0.01$ or $P<0.05$ ).

\section{Discussion}

Diverse research has revealed that estrogen can exert some effects on bone and mesenchymal stem cells (MSCs). Exogenous estrogen can not only increase bone mineral density and promote bone formation, but also has beneficial effects on the proliferation and differentiation of BMMSCs [16,22-25]. Even adipose tissue-derived stem cells (ADSCs) can possess osteogenic differentiation potential after estrogen stimulation, with greater nodule formation and mineral deposition [26]. Estrogen has also been reported to increase ALP activity, the expression of osteocalcin and mineralized nodules in PDLSCs [27]. Our previous study revealed that exogenous estrogen at physiological concentrations can enhance the odonto/ osteogenic differentiation of DPSCs [8].

In the present study, a higher concentration of E2 $\left(10^{-5} \mathrm{M}\right)$ significantly inhibited the proliferation of SCAP while $10^{-7}$ and $10^{-9} \mathrm{M}$ E2 had no effect on cell growth, indicating that there may exist a dose-dependent manner of E2 in regulating the proliferation of SCAP. To evaluate the effects of E2 on the differentiation of SCAP under similar conditions, impacts of E2 on cell proliferation should be excluded. E2 at $10^{-7} \mathrm{M}$ did not have any negative influence on cell morphology and cell proliferation as indicated by MTT, FCM and colony forming assay, while this concentration of E2 can trigger the differentiation of SCAP. Thus, $10^{-7} \mathrm{M} \mathrm{E2}$ was selected as the optimal 


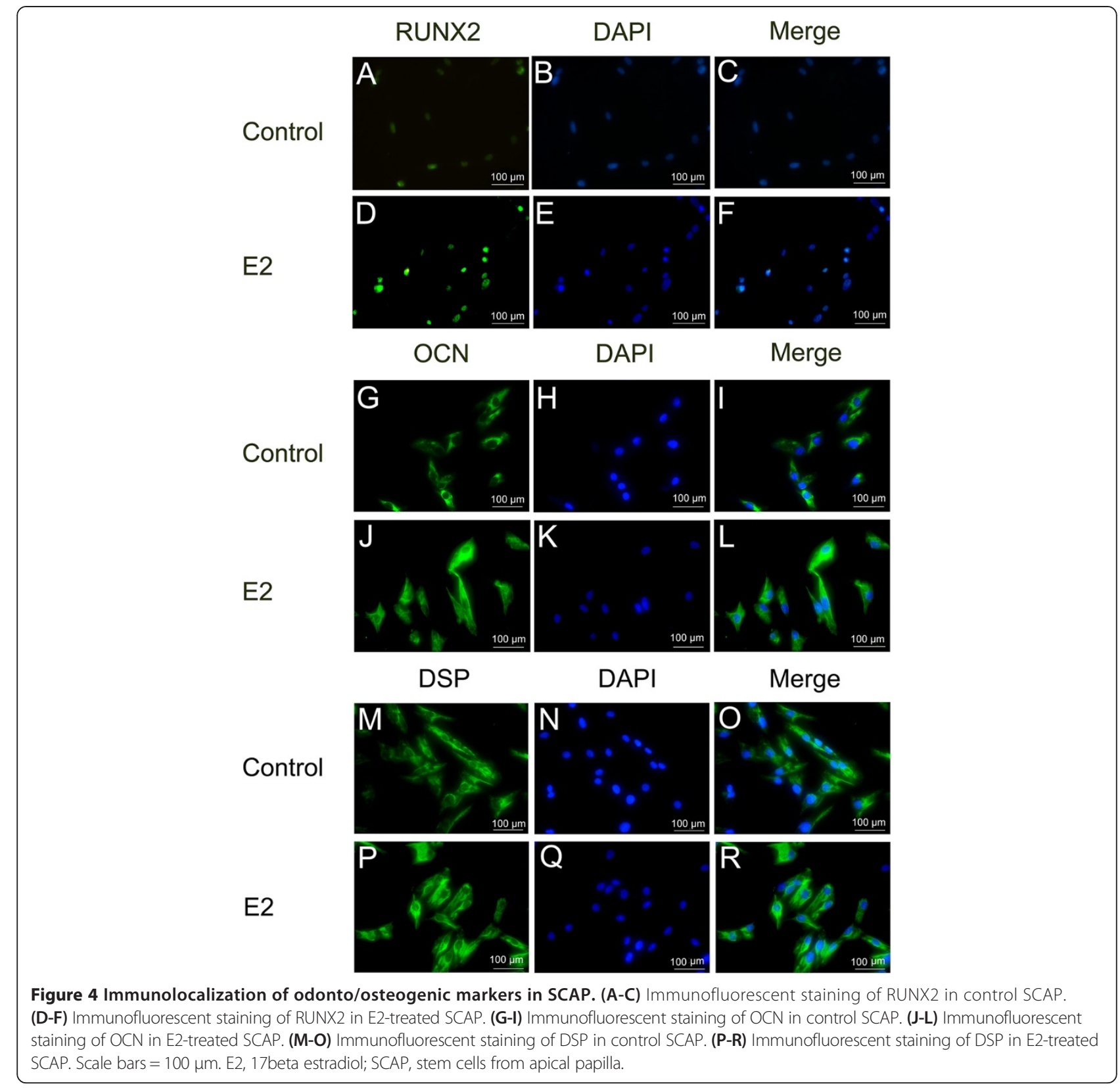

concentration to stimulate the differentiation of SCAP in vitro.

After E2 treatment, ALP activity, mineralization capacity and odonto/osteogenic markers (ALP, DMP1/DMP1, $D S P P / \mathrm{DSP}$, RUNX2/RUNX2, OSX/OSX and OCN/OCN) in SCAP were significantly upregulated. DSP protein and DSPP mRNA are tooth-specific markers that contribute to the formation of dentin and they have been reported to be expressed only in secretory odontoblasts [28-30]. DSP protein and DSPP mRNA have been identified as latestage markers of odontoblasts, the expression of which indicates the mature differentiation of odontoblasts [31]. DMP1 is expressed in odontoblasts and secretes matrix proteins to form dentin, which contributes to later dentinogenesis during postnatal development [32]. Moreover, research has suggested that DSPP is a downstream effector molecule of $D M P 1$ which is probably regulated by $D M P 1$ during dentinogenesis [32,33]. ALP, RUNX2 and OSX are early-stage markers of osteoblastic differentiation while OCN is related to the late-stage osteoblastic differentiation [24,34-36]. ALP is expressed early in the developing osteoblast, during the phase of matrix deposition and is downregulated in calcifying osteoblasts [26]. RUNX2 is a crucial factor in osteoblast and odontoblast differentiation, regulating the expression of a variety of bone-/tooth-related genes [29]. Previous studies 


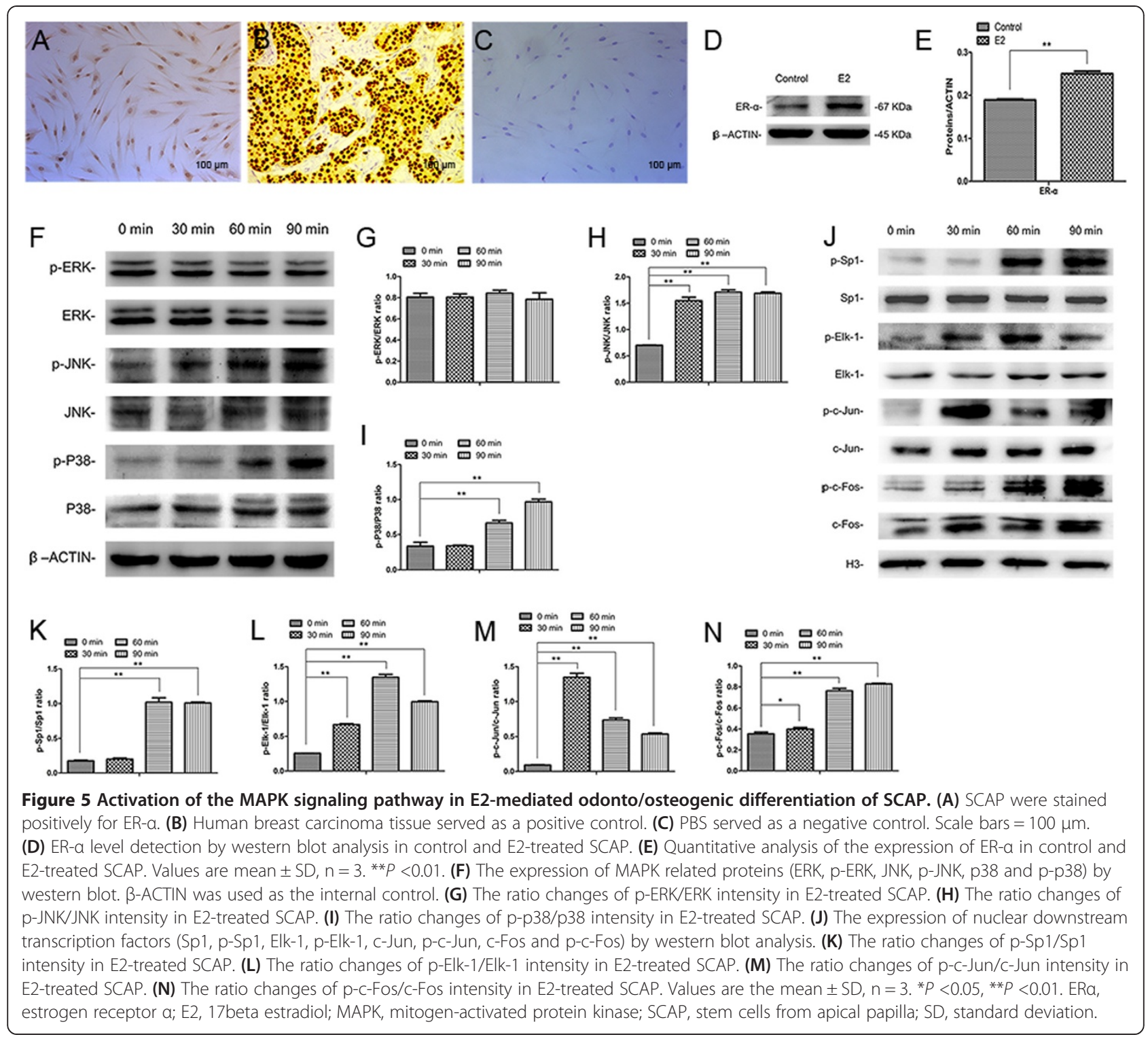

have revealed that Runx2-deficient mice have impaired tooth formation while $R U N X 2$ overexpression induces new bone generation $[37,38]$. OSX is a downstream gene of RUNX2, which plays an important role in osteogenic differentiation and bone formation and is expressed in functional odonto/osteoblasts $[8,39]$. OCN is considered a marker for bone formation in the late stage, which has a close relationship with bone maturation [40]. In the present study, both early-stage and late-stage odonto/ osteogenic markers were upregulated, implying the longterm effects of E2 on the differentiation of SCAP.

Previous research has revealed that estrogen stimulates sequential differentiation of osteoblasts and increases calcium deposition in cultured BMSSCs in an estrogen receptor (ER)-dependent manner [41]. As the classic steroid receptors, ERs (including ER- $\alpha$ and ER- $\beta$ ) generally mediate the biological responses to estrogen via genomic and non-genomic effects [42]. It is suggested that the concentration of ERs is an important determinant of cellular responsiveness to estrogen [43-45]. Moreover, an increased level of ER has been detected during the osteoblastic differentiation of rat calvarial osteoblasts [46]. In this study, E2-treated SCAP showed higher expression of ER- $\alpha$ and enhanced odonto/osteogenic potential. Recent studies have suggested that estrogen's effects on various tissues may be mediated by different cell signaling pathways [47]. E2 can regulate the bioactivity of ER$\alpha$-positive endometrial cancer cells on the basis of the MAPK signaling pathway. MAPK are an important family of protein kinases involved in transmitting signals from the cell membrane to the nucleus [48]. There are three well-characterized subfamilies of MAPK, that is, 


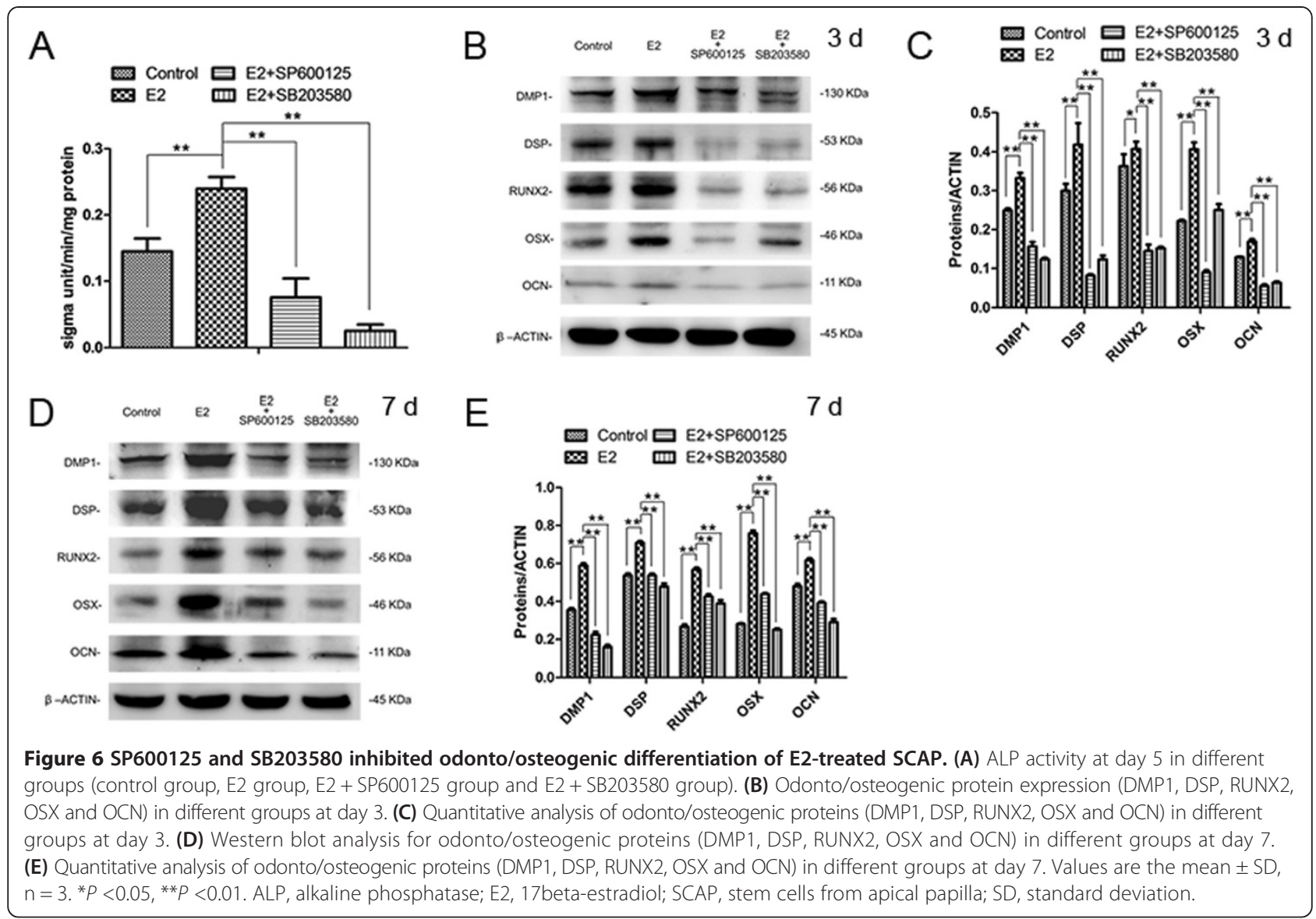

extracellular signal-regulated kinases (ERK), c-Jun Nterminal kinase (JNK) and p38 MAPK. MAPK signaling regulates cell proliferation, differentiation, survival and apoptosis in a variety of cells. In this study, E2 has some effects on the expression of phosphor-JNK and phosphorp38 but did not change the level of phosphor-ERK, indicating the activation of JNK signaling and p38 MAPK signaling. In addition, the decreased level of mineralization and differentiation in E2-treated SCAP co-cultured with SP600125 or SB203580 confirmed the potential functions of JNK and p38 MAPK signaling. Therefore, it can be concluded that E2 regulates the odonto/osteogenic differentiation of SCAP via activation of the JNK and p38 MAPK pathways.

To further evaluate the MAPK signaling involved in E2-mediated differentiation of SCAP, the nuclear downstream transcription factors of MAPK were investigated by western blot analysis. The phosphorylation of all related transcription factors (phosphor-Sp1, phosphor-Elk-1, phosphor-c-Jun and phosphor-c-Fos) can be detected in a time-dependent manner, indicating the activation of these downstream factors by E2 stimulation. E2 action occurs via the classical genomic and non-genomic ways [49]. The genomic pathway is a slow progress mediated through ERs by binding to an estrogen-responsive element (ERE) in the promotor region of different target genes [50], whereas the non-genomic action of E2 mediated by a membrane-associated receptor ( $\mathrm{MER}$ ) is very fast [51]. It is suggested that liganded ERs can not only bind directly to ERE to regulate the transcription of genes, but also are tethered to DNA by interacting with other transcription factors, such as Sp1 and activating protein-1 (AP-1) to influence the gene expression, which means E2 can induce the expression of related genes through ER/Sp1 and ER/AP-1 complexes [52,53]. In addition, E2 can trigger rapid effects including activation of a sequential series of kinases and phosphatases through membranes, such as the MAPK signaling pathway, and then induce the phosphorylation of ERs in the nuclei to regulate transcription [42,54]. As is known, when activated by upstream factors, JNK can stimulate the phosphorylation of c-Jun, its major downstream substrate, which can interact with c-Fos to form a complex, leading to enhanced AP-1 and upregulated transcription activity [55]. In addition, the activation of p38 MAPK also contributes to the formation of AP-1 by producing c-Jun and c-Fos [52]. Moreover, JNK activation can induce the phosphorylation of Elk-1 and promote subsequent transcription activities. Interestingly, E2 activated JNK and p38 MAPK pathways, but had no effect on the ERK 
pathway in this study. However, the downstream factors of all three of these pathways were activated by E2. This indicates that these downstream transcription factors can not only be activated by their upstream kinases, such as ERK, JNK and p38 MAPK, but can also be triggered by ligand-bound ERs directly. Therefore, we speculate that E2 may play an important role during the crosstalk between the ER and MAPK signaling pathways.

In this study, E2 can promote the odonto/osteogenic differentiation of SCAP. Thus, E2 can be used to stimulate the differentiation of SCAP in the apical papillae suffering from pulp infections to recover the interrupted root development. Moreover, when cultured with PDLSCs together in vivo, they can form a biological root (bio-root) as previously reported [7]. Recent studies also reported that E2 can enhance the proliferation and differentiation of PDLSCs [15]. Thus, when the two kinds of cells are cocultured with E2, they may generate a bio-root more regularly and efficiently. However, tooth growth is a complicated process that is affected by a series of factors, including patients' conditions, cell activity, growth factors and so on. More work is required to make SCAP and E2 better applied in future clinical practice.

\section{Conclusions}

In conclusion, E2 enhanced the odonto/osteogenic differentiation of SCAP via activation of the MAPK signaling pathway. These findings provide a new insight into the use of E2 for tooth engineering. More work has to be performed to explore other potential mechanisms involved in the differentiation of E2-treated SCAP, which may help the application of E2 in future endodontic practice.

\section{Abbreviations}

ADSCs: adipose tissue-derived stem cells; AGS: absorbable gelatin sponges; ALP: alkaline phosphatase; AP-1: activating protein-1; BMMSCs: bone marrow mesenchymal stem cells; CPC: cetylpyridinium chloride; DMP1: dentin matrix protein 1; DMSO: dimethyl sulfoxide; DPSCs: dental pulp stem cells; DSP: dentin sialoprotein; DSPP: dentin sialophosphoprotein; E2: 17beta-estradiol; ER: estrogen receptor; ERE: estrogen response elements; ERK: extracellular signal-regulated kinase; FBS: fetal bovine serum; FCM: flow cytometry; $\mathrm{H}$ \& E: hematoxylin and eosin; JNK: c-Jun N-terminal kinase; MAPK: mitogen-activated protein kinase; MM: mineralization-inducing media; MSCs: mesenchymal stem cells; MTT: methyl-thiazolyl-tetrazolium; OCN: osteocalcin; OSX: osterix

PBS: hosphate-buffered solution; PDLSCs: periodontal ligament stem cells; PFA: paraformaldehyde; RT-PCR: reverse transcription polymerase chain reaction; RUNX2: runt-related transcription factor 2; SCAP: stem cells while stem cells from apical papilla; a-MEM: alpha minimum essential medium.

\section{Competing interests}

The authors declare that they have no competing interests.

\section{Authors' contributions}

YL made substantial contributions to the conception and design of the study, carried out the study, performed the experiments and drafted the manuscript. MY participated in the design of the study, collected all data, performed data analysis and helped to draft the manuscript. ZLW performed the immunofluorescence, immunocytochemical and immunohistochemical staining and drafted the manuscript. YYZ contributed to the animal experiments and helped to draft the manuscript. JJL was responsible for cell culture experiments and manuscript drafting. SM and GXL contributed to mesenchymal stem cell isolation and expansion and helped to draft the manuscript. JHY conceived the study, and contributed to study design and coordination, analysis and interpretation of data, and manuscript revision. All authors read and approved the final manuscript.

\section{Acknowledgements}

This work was supported by the National Natural Science Foundation of China (No. 81371144), the Natural Science Foundation of Jiangsu Province (No. BK20131392), and the Priority Academic Program Development of Jiangsu Higher Education Institutions (PAPD, No. 2014-37).

\section{Author details}

'Department of Stomatology, Nanjing Integrated Traditional Chinese and Western Medicine Hospital Affiliated with Nanjing University of Chinese Medicine, Nanjing, Jiangsu 210014, China. ${ }^{2}$ Key Laboratory of Oral Diseases of Jiangsu Province and Stomatological Institute of Nanjing Medical University, 140 Hanzhong Road, Nanjing, Jiangsu 210029, China. ${ }^{3}$ Endodontic Department, School of Stomatology, Nanjing Medical University, 136 Hanzhong Road, Nanjing, Jiangsu 210029, China.

Received: 13 February 2014 Revised: 28 October 2014

Accepted: 29 October 2014 Published: 17 November 2014

\section{References}

1. Huang GT: A paradigm shift in endodontic management of immature teeth: conservation of stem cells for regeneration. J Dent 2008, 36:379-386.

2. Huang GT, Sonoyama W, Liu Y, Liu H, Wang S, Shi S: The hidden treasure in apical papilla: the potential role in pulp/dentin regeneration and BioRoot engineering. J Endod 2008, 34:645-651.

3. Huang GT: Apexification: the beginning of its end. Int Endod J 2009, 42:855-866.

4. Sonoyama W, Liu Y, Yamaza T, Tuan RS, Wang S, Shi S, Huang GT: Characterization of the apical papilla and its residing stem cells from human immature permanent teeth: a pilot study. J Endod 2008, 34:166-171

5. Sonoyama W, Liu Y, Fang D, Yamaza T, Seo BM, Zhang C, Liu H, Gronthos S, Wang CY, Wang S, Shi S: Mesenchymal stem cell-mediated functional tooth regeneration in swine. PLoS One 2006, 1:e79.

6. Abe S, Yamaguchi S, Watanabe A, Hamada K, Amagasa T: Hard tissue regeneration capacity of apical pulp derived cells (APDCs) from human tooth with immature apex. Biochem Biophys Res Commun 2008, 371:90-93.

7. Dadu SS: Tooth regeneration: current status. Indian J Dent Res 2009, 20:506-550.

8. Wang $Y$, Zheng $Y$, Wang Z, Li J, Zhang G, Yu J: 10(-7) m17 3 -oestradiol enhances odonto/osteogenic potency of human dental pulp stem cells by activation of the NF-KB pathway. Cell Prolif 2013, 46:677-684.

9. Bakopoulou A, Leyhausen G, Volk J, Koidis P, Geurtsen W: Comparative characterization of STRO-1neg/CD146pos and STRO-1 pos/CD146pos apical papilla stem cells enriched with flow cytometry. Arch Oral Biol 2013, 58:1556-1568.

10. Ruparel NB, Teixeira FB, Ferraz CC, Diogenes A: Direct effect of intracanal medicaments on survival of stem cells of the apical papilla. J Endod 2012, 38:1372-1375

11. Jiang Q, Du J, Yin X, Shan Z, Ma Y, Ma P, Fan Z: Shh signaling, negatively regulated by BMP signaling, inhibits the osteo/dentinogenic differentiation potentials of mesenchymal stem cells from apical papilla. Mol Cell Biochem 2013, 383:85-93.

12. Wang $Y$, Yan M, Yu Y, Wu J, Yu J, Fan Z: Estrogen deficiency inhibits the odonto/osteogenic differentiation of dental pulp stem cells via activation of the NF-KB pathway. Cell Tissue Res 2013, 352:551-559.

13. Xue $P$, Wang $Y$, Yang J, Li Y: Effects of growth hormone replacement therapy on bone mineral density in growth hormone deficient adults: a meta-analysis. Int J Endocrinol 2013, 2013:1-13.

14. Bin Zhang YL, Zhou Q, Ding Y: Estrogen deficiency leads to impaired osteogenic differentiation of periodontal ligament stem cells in rats. Tohoku J Exp Med 2011, 223:177-186.

15. Mamalis A, Markopoulou C, Lagou A, Vrotsos I: Oestrogen regulates proliferation, osteoblastic differentiation, collagen synthesis and periostin gene expression in human periodontal ligament cells through oestrogen receptor beta. Arch Oral Biol 2011, 56:446-455. 
16. Zhang M, Chen FM, Wang AH, Chen YJ, Lv X, Wu S, Zhao RN: Estrogen and its receptor enhance mechanobiological effects in compressed bone mesenchymal stem cells. Cells Tissues Organs 2012, 195:400-413.

17. Zhao JW, Gao ZL, Mei H, Li YL, Wang Y: Differentiation of human mesenchymal stem cells: the potential mechanism for estrogen-induced preferential osteoblast versus adipocyte differentiation. Am J Med Sci 2011, 341:460-468.

18. Yu J, Wang Y, Deng Z, Tang L, Li Y, Shi J, Jin Y: Odontogenic capability: bone marrow stromal stem cells versus dental pulp stem cells. Biol Cell 2007, 99:465-474.

19. Lei G, Yan M, Wang Z, Yu Y, Tang C, Yu J, Zhang G: Dentinogenic capacity: immature root papilla stem cells versus mature root pulp stem cells. Biol Cell 2011, 103:185-196.

20. Wang S, Mu J, Fan Z, Yu Y, Yan M, Lei G, Tang C, Wang Z, Zheng Y, Yu J, Zhang G: Insulin-like growth factor 1 can promote the osteogenic differentiation and osteogenesis of stem cells from apical papilla. Stem Cell Res 2012, 8:346-356.

21. Yu J, He H, Tang C, Zhang G, Li Y, Wang R, Shi J, Jin Y: Differentiation potential of STRO-1+ dental pulp stem cells changes during cell passaging. BMC Cell Biol 2010, 11:32.

22. Galea GL, Price JS, Lanyon LE: Estrogen receptors' roles in the control of mechanically adaptive bone (re)modeling. Bonekey Rep 2013, 2:413.

23. Ozono S, Fujita T, Matsuo M, Todoki K, Ohtomo T, Negishi H, Kawase T: Co-treatment with basic fibroblast growth factor and 17beta-estradiol in the presence of dexamethasone accelerates bone formation by rat bone marrow stromal cell culture. Nihon Hotetsu Shika Gakkai Zasshi 2008, 52:366-374.

24. Gopalakrishnan V, Vignesh RC, Arunakaran J, Aruldhas MM, Srinivasan N: Effects of glucose and its modulation by insulin and estradiol on BMSC differentiation into osteoblastic lineages. Biochem Cell Biol 2006, 84:93-101.

25. Hong L, Colpan A, Peptan IA: Modulations of $17-\beta$ estradiol on osteogenic and adipogenic differentiations of human mesenchymal stem cells. Tissue Eng 2006, 12:2747-2753.

26. Taskiran D, Evren V: Stimulatory effect of $17 \beta$-estradiol on osteogenic differentiation potential of rat adipose tissue-derived stem cells. Gen Physiol Biophys 2011, 30:167-174.

27. Liang L, Yu JF, Wang Y, Wang G, Ding Y: Effect of estrogen receptor beta on the osteoblastic differentiation function of human periodontal ligament cells. Arch Oral Biol 2008, 53:553-557.

28. lejima D, Sumita $Y$, Kagami $H$, Ando $Y$, Ueda M: Odontoblast marker gene expression is enhanced by a CC-chemokine family protein MIP-3a in human mesenchymal stem cells. Arch Oral Biol 2007, 52:924-931.

29. Chen S, Gluhak-Heinrich J, Wang YH, Wu YM, Chuang HH, Chen L, Yuan GH, Dong J, Gay I, MacDougall M: Runx2, osx, and dspp in tooth development. J Dent Res 2009, 88:904-909.

30. Yamazaki H, Kunisada T, Miyamoto A, Tagaya H, Hayashi SI: Tooth-specific expression conferred by the regulatory sequences of rat dentin sialoprotein gene in transgenic mice. Biochem Biophys Res Commun 1999, 260:433-440.

31. Yamakoshi Y: Dentinogenesis and Dentin Sialophosphoprotein (DSPP). J Oral Biosci 2009, 51:134

32. Ye L, MacDougall M, Zhang S, Xie Y, Zhang J, Li Z, Lu Y, Mishina Y, Feng JQ: Deletion of dentin matrix protein-1 leads to a partial failure of maturation of predentin into dentin, hypomineralization, and expanded cavities of pulp and root canal during postnatal tooth development. J Biol Chem 2004, 279:19141-19148.

33. Gibson MP, Zhu Q, Wang S, Liu Q, Liu Y, Wang X, Yuan B, Ruest LB, Feng JQ, D'Souza RN, Qin C, Lu Y: The rescue of dentin matrix protein 1 (DMP1)-deficient tooth defects by the transgenic expression of dentin sialophosphoprotein (DSPP) indicates that DSPP is a downstream effector molecule of DMP1 in dentinogenesis. J Biol Chem 2013, 288:7204-7214

34. Komori T: Regulation of osteoblast differentiation by Runx2. Adv Exp Med Biol 2010, 658:43-49.

35. Ni P, Fu S, Fan M, Guo G, Shi S, Peng J, Luo F, Qian Z: Preparation of poly (ethylene glycol)/polylactide hybrid fibrous scaffolds for bone tissue engineering. Int J Nanomedicine 2011, 6:3065-3075.

36. Wade-Gueye NM, Boudiffa M, Vanden-Bossche A, Laroche N, Aubin JE, Vico L, Lafage-Proust MH, Malaval L: Absence of bone sialoprotein (BSP) impairs primary bone formation and resorption: the marrow ablation model under PTH challenge. Bone 2012, 50:1064-1073.
37. D'Souza RN, Aberg T, Gaikwad J, Cavender A, Owen M, Karsenty G, Thesleff I: $\mathrm{Cbfa} 1$ is required for epithelial-mesenchymal interactions regulating tooth development in mice. Development 1999, 126:2911-2920.

38. Takahashi T: Overexpression of Runx2 and MKP-1 stimulates transdifferentiation of 3 T3-L1 preadipocytes into bone-forming osteoblasts in vitro. Calcif Tissue Int 2011, 88:336-347.

39. Baek WY, Lee MA, Jung JW, Kim SY, Akiyama H, de Crombrugghe B, Kim JE: Positive regulation of adult bone formation by osteoblast-specific transcription factor osterix. J Bone Miner Res 2009, 24:1055-1065.

40. Neugebauer BM, Moore MA, Broess M, Gerstenfeld LC, Hauschka PV: Characterization of structural sequences in the chicken osteocalcin gene: expression of osteocalcin by maturing osteoblasts and by hypertrophic chondrocytes in vitro. J Bone Miner Res 1995, 10:157-163.

41. Qu Q, Perala-Heape M, Kapanen A, Dahllund J, Salo J, Vaananen HK, Harkonen P. Estrogen enhances differentiation of osteoblasts in mouse bone marrow culture. Bone 1998, 22:201-209.

42. Heldring N, Pike A, Andersson S, Matthews J, Cheng G, Hartman J, Tujaque M, Strom A, Treuter E, Warner M, Gustafsson JA: Estrogen receptors: how do they signal and what are their targets. Physiol Rev 2007, 87:905-931.

43. Kuiper GG, Enmark E, Pelto-Huikko M, Nilsson S, Gustafsson JA: Cloning of a novel receptor expressed in rat prostate and ovary. Proc Natl Acad SCi U S A 1996, 93:5925-5930.

44. Tremblay GB, Tremblay A, Copeland NG, Gilbert DJ, Jenkins NA, Labrie F, Giguere V: Cloning, chromosomal localization, and functional analysis of the murine estrogen receptor beta. Mol Endocrinol 1997, 11:353-365.

45. Santagati S, Gianazza E, Agrati P, Vegeto E, Patrone C, Pollio G, Maggi A: Oligonucleotide squelching reveals the mechanism of estrogen receptor autologous down-regulation. Mol Endocrinol 1997, 11:938-949.

46. Bodine PV, Henderson RA, Green J, Aronow M, Owen T, Stein GS, Lian JB, Komm BS: Estrogen receptor-alpha is developmentally regulated during osteoblast differentiation and contributes to selective responsiveness of gene expression. Endocrinology 1998, 139:2048-2057.

47. Kousteni S, Han L, Chen JR, Almeida M, Plotkin LI, Bellido T, Manolagas SC: Kinase-mediated regulation of common transcription factors accounts for the bone-protective effects of sex steroids. J Clin Invest 2003, 111:1651-1664.

48. Migliaccio A, Zhou C, Steplowski TA, Dickens HK, Malloy KM, Gehrig PA, Boggess JF, Bae-Jump VL: Estrogen induction of telomerase activity through regulation of the mitogen-activated protein kinase (MAPK) dependent pathway in human endometrial cancer cells. PLoS One 2013, 8:e55730.

49. Derwahl M, Nicula D: Estrogen and its role in thyroid cancer. Endocr Relat Cancer 2014, 21:T273-T283.

50. Nilsson S, Makela S, Treuter E, Tujague M, Thomsen J, Andersson G, Enmark E, Pettersson K, Warner M, Gustafsson JA: Mechanisms of estrogen action. Physiol Rev 2001, 81:1535-1565.

51. Moriarty K, Kim KH, Bender JR: Minireview: estrogen receptor-mediated rapid signaling. Endocrinology 2006, 147:5557-5563.

52. Saville B, Wormke M, Wang F, Nguyen T, Enmark E, Kuiper G, Gustafsson JA, Safe S: Ligand-, cell-, and estrogen receptor subtype (alpha /beta )-dependent activation at GC-rich (Sp1) promoter elements. J Biol Chem 2000, 275:5379-5387.

53. Babu RL, Naveen Kumar M, Patil RH, Devaraju KS, Ramesh GT, Sharma SC Effect of estrogen and tamoxifen on the expression pattern of AP-1 factors in MCF-7 cells: role of c-Jun, c-Fos, and Fra-1 in cell cycle regulation. Mol Cell Biochem 2013, 380:143-151.

54. Xiao HH, Gao QG, Zhang Y, Wong KC, Dai Y, Yao XS, Wong MS: Vanillic acid exerts oestrogen-like activities in osteoblast-like UMR 106 cells through MAP kinase (MEK/ERK)-mediated ER signaling pathway. J Steroid Biochem Mol Biol 2014, 144 Pt B:382-391.

55. Tang YQ, Jaganath I, Manikam R, Sekaran SD: Phyllanthus suppresses prostate cancer cell, PC-3, proliferation and induces apoptosis through multiple signalling pathways (MAPKs, PI3K/Akt, NFkappaB, and hypoxia). Evid Based Compl Alternat Med 2013, 2013:609581.

\section{doi:10.1186/scrt515}

Cite this article as: Li et al:: 17beta-estradiol promotes the odonto/osteogenic differentiation of stem cells from apical papilla via mitogen-activated protein kinase pathway. Stem Cell Research \& Therapy 2014 5:125. 